\title{
Quantitative X-ray Microanalysis of Multi-layered Specimens: Capability and Accuracy.
}

\author{
C. Merlet
}

GM, CNRS, Université de Montpellier II, Pl. E. Bataillon, 34095 Montpellier cedex 5, France

The determination of the thickness and composition of thin layer and multilayer deposited on substrates is an important area in semiconductor research and thin-film technology. Electron probe microanalysis (EPMA), originally developed to determine the composition of the bulk samples at the micron scale, has become a well established technique to determine the compositions and the thickness of thin multilayer deposited on a substrate [1-7]. This technique can be used to determine the film thickness in a range of a few micrometers to a monolayer. Measurements of thin-layer thicknesses can also be performed by some others techniques such as Rutherford backscattering spectrometry (RBS), Atomic Force Microscopy (AFM), Secondary Ion Mass spectrometry (SIMS), optical interferometry (OI), x-ray fluorescence XRF etc. However in comparison to the other techniques and beyond the microscopic aspect of the method, and also the fact that it is a nondestructive technique, one of the advantages of the EPMA method is the opportunity to have simultaneously the layer thickness and the composition with accuracies similar to the one obtained on bulk sample. In addition, the equipment is available in many laboratories, i.e., a scanning electron microscope with an Energy Dispersive spectrometer (EDS) or more rarely an electron probe with Wavelength Dispersive spectrometers (WDS).

The thin-multilayer method by EPMA is based on the comparison of the ratios of $x$-ray intensities (k-ratio) emitted by the elements of the various layers to those emitted from bulk standards under same experimental conditions. By varying the energy of the incident electrons, and thus the excitation depths, the different layers can be analyzed. Typical incident electrons energies of 5-40 $\mathrm{keV}$ have excitation depths from $0.2-10 \mu \mathrm{m}$. For an element of a thin layer at the surface, an increase of the energy of the incident electrons increases the excitation depths and as a result induces a decrease of the k-ratio. Similarly for a buried layer, an increase in the energy of the incident electrons results in an increase followed by a decrease of the k-ratio. The observed variation of the k-ratio with incident electron energy of the layers and of the substrate is the input of the EPMA quantification code, which determines the thickness of the layers by fitting the experimental k-ratios with a Monte Carlo (MC) simulation code [5,6] or with an analytical x-ray emission model [1-4]. The accuracy of the result is therefore directly dependent on the accuracy of the theoretical model. Since quantitative results are obtained with the help of numerical iterative procedures or with a manual trial and error approach, in practice, only analytical models are used. Monte Carlo (MC) simulation code, not always more accurate [7], but very time consuming, are generally used to optimize or assess analytical models.

To convert the measured k-ratio from elements of the layers in thicknesses and compositions, an analytical x-ray emission model requires an accurate description of the x-ray depth distribution $(\phi(\rho z))$ from which the emitted $\mathrm{x}$-ray intensities are calculated. It is worth noting that, for each element corresponds a specific $(\phi(\rho z))$ distribution which varies with incident electrons energy. However, a multilayer x-ray model is much more complex than for a bulk x-ray model, because discontinuities exist at the layers interface and an analytical model must take into account these 
various aspects. Analytical $(\phi(\rho z))$ models, which have been successfully used in many applications, are known to be less accurate when there are large differences in atomic number between the different layers. In analytical approaches, in a stratified sample and for each element of layers, the $\phi(\rho z)$ distributions are built by using a weighting procedure according to the specific layers combinations. The simplest case corresponds to a thin layer/substrate with pure elements whose atomic numbers are neighboring. For this simple case the electron scattering and the x-ray generation are similar as in bulk sample and the $\phi(\rho z)$ distributions from thin layer/substrate are similar to bulk sample. When the deviation of the atomic number between thin layer and substrate increases, the difference in electron scattering properties between thin layer and substrate increases and consequently a change in the $\phi(\rho z)$ distributions comparatively to bulk samples. Similarly the thickness of the thin layer relatively to the $\phi(\rho z)$ distributions can vary between two extremes: extremely thin or extremely thick. In the first case, the $\phi(\rho z)$ distributions from layer/substrate correspond to a bulk with the composition of the substrate, and in the second case, to a bulk with the composition of the layer. In the intermediate cases the $\phi(\rho z)$ distributions vary between these two extremes.

Depending of the instrument (EDS or WDS), the operator experience, and the sample structure, we can be expected with thin-multilayer method by EPMA an uncertainty in the thickness determination less than $10 \%[1,7]$ even when there are large differences in atomic number between the different layers. This technique is more reliable for the concentration because the uncertainty of the thickness is the same for all elements of a layer and as a result, in best cases, accuracy similar to bulk samples can be achieved. In the determination, a buried layer or a very thin layer will be often less accurate than a surface layer or a thicker layer. In a general way, in addition to the uncertainty of intensity measurement and of the fitting routine, specific deviations caused by the $\phi(\rho z) \operatorname{method}$ must be added. Indeed, calculations require the knowledge of many atomic parameters that describe the electron interaction and the x-ray emission, and mainly the ionization cross section and the mass absorption coefficient. In thin layers when using standards and unlike bulk samples, uncertainties in these atomic parameters are less compensated. Moreover, the thin multilayer method requires a hypothesis concerning the sample structure (number of layers, elements in each of the layers) and consequently, the operator experience is crucial for the quality of the results.

\section{References}

[1] GF Bastin and HJM Heijligers, X-ray spectrometry 29 (2000) 212.

[2] J.L. Pouchou and F. Pichoir. In Electron Probe Quantitation, Edited by K.F.J. Heinrich and D. Newbury, plenum Press, New York, (1991) 31.

[3] C. Merlet, Proc. of Microbeam Analysis, Edited by E. S. Etz, VCH Publishers, (1995) 203.

[4] X. Llovet and C. Merlet, Micros. Microanal. 16, (2010) 21.

[5] F. Salvat et al., PENELOPE. A Code System for Monte Carlo Simulation of Electron and Photon Transport, OECD/Nuclear Energy Agency, Issy-les-Moulineaux, France, (2009).

[6] D. Drouin et al. Scanning 29, 3 (2007) 92.

[7] P. Statham et al. IOP Conf. Series: Materials Science and Engineering 32 (2012) 012024. 